\title{
Laser-induced break-up of water jet waveguide
}

\author{
P. Couty, Á. Spiegel, N. Vágó, B. I. Ugurtas, P. Hoffmann
}

Abstract In this article, an optical method to control the break-up of high-speed liquid jets is proposed. The method consists of focusing the light of a pulsed laser source into the jet behaving as a waveguide. Experiments were performed with the help of a Q-switched frequency doubled Nd:Yag laser $(\lambda=532 \mathrm{~nm})$. The jet diameter was $48 \mu \mathrm{m}$ and jet velocities from 100 to $200 \mathrm{~m} / \mathrm{s}$. To study the laser-induced water jet break-up, observations of the jet coupled with the high power laser were performed for variable coupling and jet velocity conditions. Experimentally determined wavelength and growth rate of the lasergenerated disturbance were also compared with the ones predicted by linear stability theory of free jets.

\section{1}

\section{Introduction}

The stability of high-speed jets has been subject of active research for more than a century. Recently, the light guiding properties of water jet have been used in the framework of laser machining (Richerzhagen 2001). In this application, high power pulsed laser light is coupled into thin water jets, with diameters between 30 and $150 \mu \mathrm{m}$, and jet speeds between 50 and $450 \mathrm{~m} / \mathrm{s}$. The advantages of this technique are the cooling effect of water as well as the management of the waste during laser processing.

Received: 26 May 2003 / Accepted: 8 December 2003

Published online: 18 March 2004

(C) Springer-Verlag 2004

P. Couty $(\varangle)$, P. Hoffmann

EPFL/STI/IOA/Advanced Photonics Laboratory,

Lausanne BM, 1015, Lausanne, Switzerland

E-mail: philippe.couty@epfl.ch

Á. Spiegel, N. Vágó

Department of Atomic Physics, Budapest University of Technology and Economics, Budafoki út 8,1111 , Budapest, Hungary

\section{B. I. Ugurtas}

EPFL/STI/IMHEF/Laboratory Fluid Mechanics,

Lausanne, 1015, Lausanne, Switzerland

The authors are grateful to the Commission for Technology and Innovation (KTI/CTI project N5314.3 KTS) for co-financing this work. The experiments have been carried out and supported by the Research and Development laboratory of the company SYNOVA SA where the Laser-Microjet ${ }^{\circledR}$ technology is developed and applied. The authors are very grateful for the collaboration of the director Bernold Richerzhagen and the head of the RD group Frank Wagner.
Liquid jets emanating from a round nozzle into quiescent gaseous atmosphere tends to break up into droplets because of the growth of initial disturbances. Depending on the characteristics of the initial disturbance, a jet can be controlled or non-controlled. Most experiments dealing with the measurement of the growth rate of the disturbance use controlled jet destabilization. The perturbation is controlled by either an electric device (Goedde and Yuen 1970; Lafrance 1975), an acoustic device (Donnelly and Glaberson 1966; Collicott et al. 1994; Rutland and Jameson 1971) or a piezoelectric transducer (Taub 1976; Xing et al. 1996). When the disturbance is forced, the jet comes to a steady state as the developed disturbance maintains a constant phase relation with the imposed disturbances. As a consequence, the break-up length is well defined within the natural uncertainty of the break-up.

The other cases are freely propagating jets without controlled disturbances. Under constant working conditions, the destabilization process changes in space and in time. This phenomenon is called break-up fluctuation. Former experiments carried out with maximum jet velocities of $30 \mathrm{~m} / \mathrm{s}$ report variations up to $30 \%$ (Grant and Middleman 1966; Sterling and Sleicher 1975; Leroux et al. 1996). Most authors characterize the stability of freely propagating jets by the mean value of the break-up length.

In this article, we propose a method to control the break up of small diameter free water jets with natural frequencies from tens of $\mathrm{kHz}$ up to $\mathrm{MHz}$. The effect of high power green laser pulses on the stability of a $48 \mu \mathrm{m}$ water jet with velocities 94 and $209 \mathrm{~m} / \mathrm{s}$ is studied. At first, the coupling of laser light into the water jet as well as the visualization system is described in the experimental setup and the possible mechanisms for the laser-induced jet break-up are described. The break-up length is measured using the so-called "light-guided method" proposed by Vágó et al. (2003). By this method, the laser is coupled in the jet waveguide and the break-up length is indirectly measured by detecting the green light scattered from the region corresponding to jet break-up point. Then, visualizations at high magnification reveal the scattering of jet break-up at low and high average power of the laser. To track the surface wave disturbances leading to the final break-up, synchronization of the laser pulse with the visualization and moving the visualization system along the jet were performed. Furthermore, we give a comparison of measured wavelength and growth rate of the lasergenerated disturbance with the ones predicted in a numerical model. Finally, observations of the scattered light for different off-center coupling are presented. 


\section{Experimental set-up}

\section{1}

\section{Generation of the jet}

The jet is produced by a sharp edged nozzle in a coupling unit (see Fig. 1). The particularity of this nozzle type is to create a separated flow, i.e., the nozzle length to diameter ratio is zero. The coupling unit is fed by a continuous water flow provided by a two-cylinder pump with an adjustable pressure range of $\Delta P$ from 0 to 1000 bar allowing jet velocity of $V_{\mathrm{j}}$ from 0 to $420 \mathrm{~m} / \mathrm{s}$. The relation between the operating pressure and the jet velocity is given in Eq. 1.

$V_{\mathrm{j}}=K \sqrt{\frac{2 \Delta P}{\rho}}$

where $K=0.94$ is the discharge coefficient of the coupling unit.

The coupling unit consists of a quartz window, a chamber, and a nozzle holder where nozzles of different diameters $D_{\text {nozzle }}$ can be mounted. The diameter of the jet is smaller than the nozzle diameter because of the vena contracta effect, according to Eq. 2.

$D_{\mathrm{j}}=\beta D_{\text {nozzle }}$

where $\beta$ is the contraction ratio of the jet. $\beta=0.8$ was measured for the nozzles used in the experiments, independently of the nozzle size and jet velocity.

\section{2}

\section{High power laser coupled into water microjet}

The laser coupling is schematically presented in Fig. 3 (zone A). A Q-switched frequency doubled Nd:YAG laser (Quantronix Corp., East Setauket, NY, USA-532CQ$100 \mathrm{~W}, \lambda=532 \mathrm{~nm}, M^{2} \simeq 35$ ) is used as light source. The repetition rate of the laser pulses $1 / \Delta t$ can be set from 10 to $20 \mathrm{kHz}$, and pulse width ranges from 96 to $317 \mathrm{~ns}$. The coupling into the jet is achieved by using a beam expander $2-8 \times$ and a lens of focal length $f=23 \mathrm{~mm}$. As illustrated in

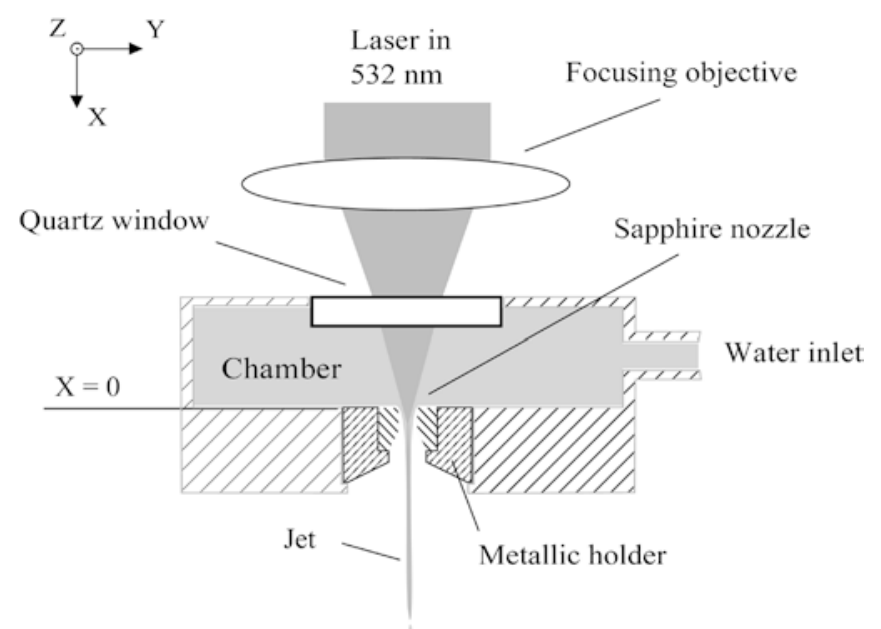

Fig. 1. Schematic of the coupling unit (not to scale)
Fig. 2, this leads to a beam waist diameter $2 w_{\mathrm{o}}\left(1 / e^{2}\right)$ of $26 \mu \mathrm{m}$ focused into the jet.

The alignment and focusing of the coupling was achieved by means of moving stages for the focusing lens ( $X$ direction, beam axis), and the coupling unit ( $Y$ and $Z$ direction). The coupling alignment of the laser beam into the water jet was obtained with the help of a CCD camera monitoring the nozzle entrance. To prevent nozzle damage during the alignment procedure, the laser light can be attenuated with the help of an attenuation block. Thus, only few $\mathrm{mW}$ are focused on the nozzle.

\section{3}

\section{Shadowgraphy of the laser-generated disturbances}

The visualization system used to observe the laser-generated disturbances is schematically presented in Fig. 3 (zone B).

This set-up is also used to observe the light scattered out of the jet waveguide. The shadowgraphy set-up consists of a light source with $0.8 \mu$ s pulse duration (full width half maxium), a collimator lens $f=46 \mathrm{~mm}$, a microscope objective $\times 10$ and a fast shutter camera (Flashcam, PCO AG, Kelheim, Germany) with a minimum exposure time of $1 \mu \mathrm{s}$. The whole shadowgraphy system is mounted on an optical rail that can be displaced to observe different parts of the jet with high magnification. Synchronization is needed as shown in Fig. 4 for triggering the image system. A diode (diode 1) is installed behind an attenuation highreflection (HR) mirror in the high power laser path. The diode signal is monitored with an oscilloscope and is used to produce a trigger signal based on the laser pulse. To scan the jet and visualize the traveling disturbance on the jet, the shadowgraphy system is moved along the jet while adapting the delay $1(d t 1)$ on the delay generator triggering the flash. The amount of overlap between the flash emission and light integration of the camera is adjusted by setting delay $2(d t 2)$. The actual exposure time is shorter than $0.8 \mu \mathrm{s}$.

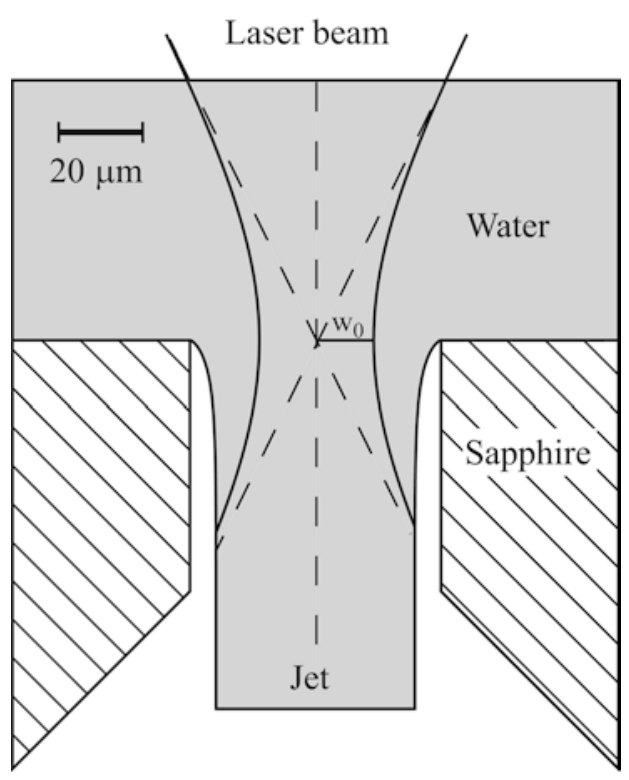

Fig. 2. Geometry of the produced jet with the $1 / e^{2}$ contours of the focused laser beam 


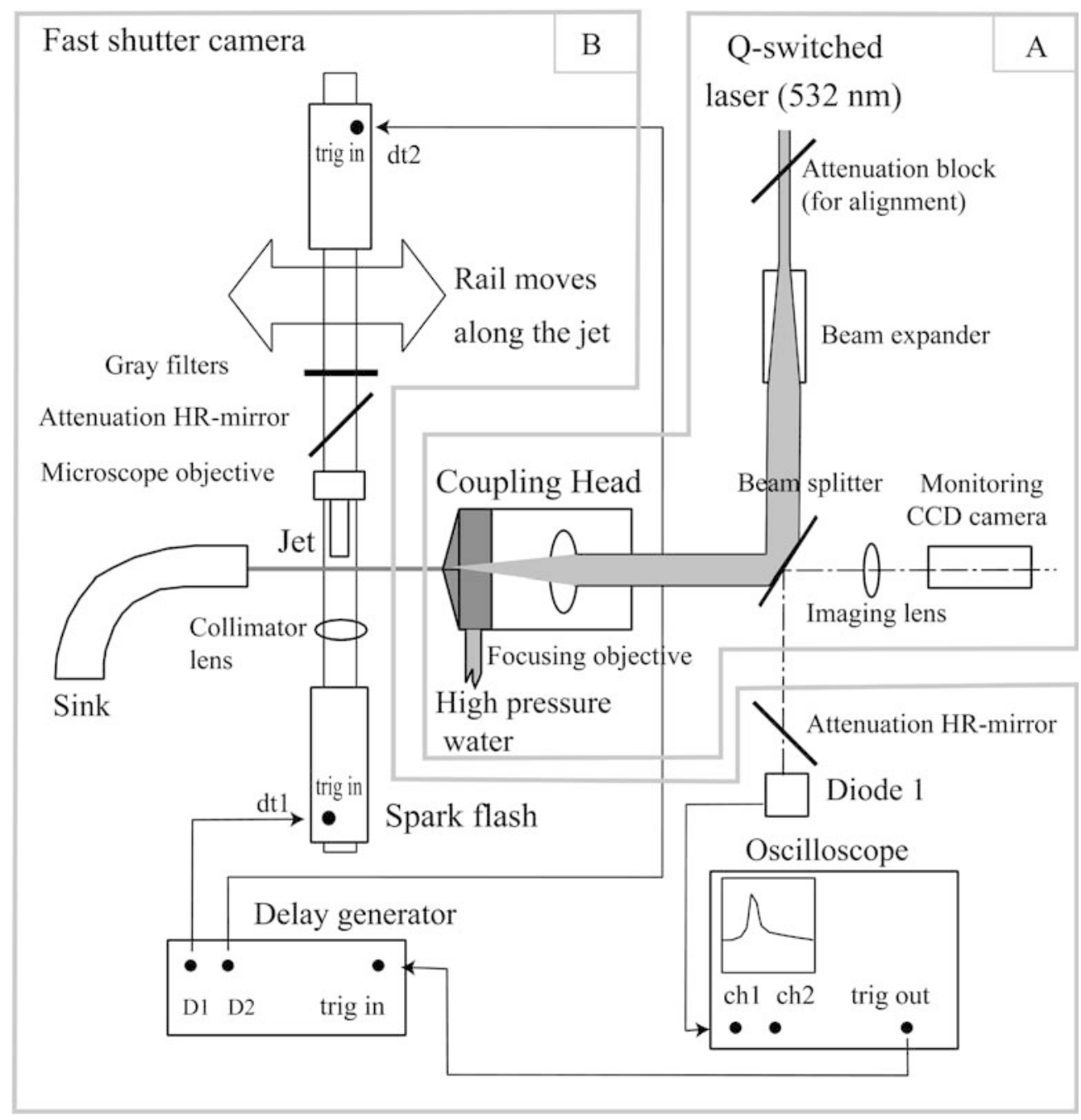

Fig. 3a, b. Schematic of the experimental set-up. a Laser beam jet coupling system. b Mobile shadowgraphy system

\section{4}

\section{Observation of scattered light along the entire jet}

Using the method presented by Vágó et al. (2003), visualizing the break up of the jet was achieved. The principle of the method can be summarized as follows. The jet behaves as a cylindrical waveguide until the point where the amplitude of surface waves is big enough to scatter out the light from the jet. Observing the jet from a direction perpendicular to its axis, the appearing light indicates the actual location of the break-up. In the present set-up, the high intensity laser pulses are both used to disturb the jet and to visualize the disturbances traveling in the jet waveguide. Low magnification observation permits to observe light scattered out from the entire jet. To scale the image, a ruler indicates the distance in $\mathrm{mm}$ from the jet origin, i.e., the inlet surface of the nozzle.

The high intensity green light is attenuated with a HR mirror and gray filters. The camera shutter is triggered on the laser pulse with minimum exposure time $1 \mu \mathrm{s}$. In this way single laser pulses are recorded for laser repetition rates from 10 to $20 \mathrm{kHz}$. Exposure times of $10 \mathrm{~ms}(10$ cycles of $1 \mathrm{~ms}$ ) are also applied for light integration of numerous laser pulses and thus statistical analysis of the jet disruption is possible. Image processing is then performed to determine the location of the light intensity peaks along the jet.

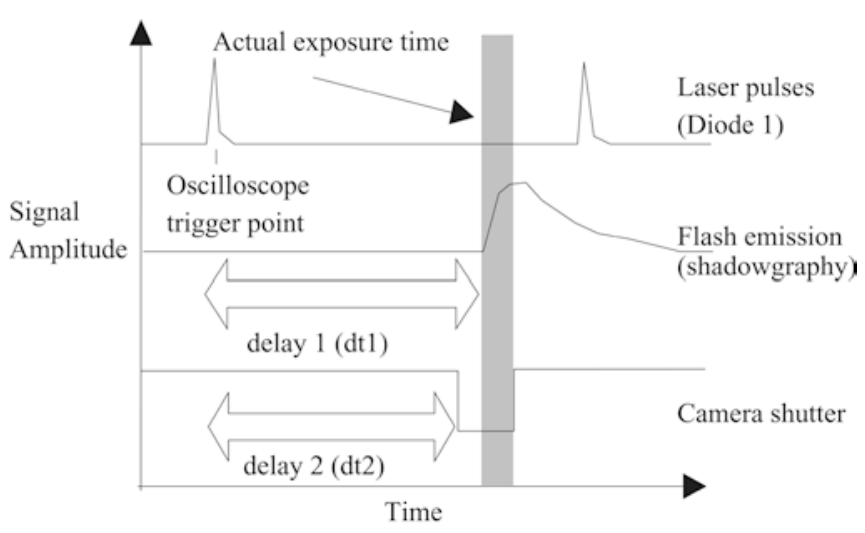

Fig. 4. Synchronization diagram of the visualization system

\section{3}

Mechanism of the laser-induced break-up

3.1

Basic principle

The laser pulse width is hundreds of nanoseconds, therefore the laser excitation is of impulse type containing high frequencies from tens of $\mathrm{kHz}$ to $\mathrm{MHz}$. This frequency range actually corresponds to the bandwidth of the natural 
frequencies of the jet. The consequence for the jet is to disrupt under the action of the disturbance with the highest growth rate (Sterling and Sleicher 1975).

\section{2}

\section{Energy coupling}

The energy transfer from the laser pulse to the jet can be explained by both the absorption of water with the high intensity pulses and the localized heating of the nozzle edge.

Although the absorption coefficient for the laser wavelength $532 \mathrm{~nm}$ in water is about $0.00041 / \mathrm{cm}$, there is a threshold for the light to be non-linearly absorbed when high intensity light is involved. Experiments report about stimulated Raman scattering (Spiegel et al. 2004) and laser-induced cavitation where optical breakdown is reached in cold water leading to the rapid growth of a water vapor bubble (Vogel et al. 1989; Noack et al. 1998).

Similarly, the sapphire nozzle can absorb the light. Transparent material such as optical crystal (sapphire, quartz, diamond, etc.) and special glass (fused silica, float glass, etc.) can be damaged by high power laser

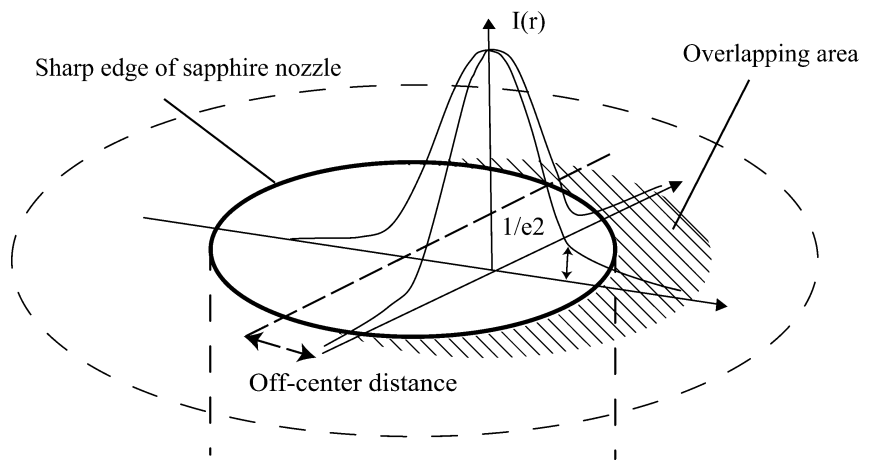

Fig. 5. Overlap of the radial intensity $I(r)$ of a gaussian beam with the nozzle for off-center coupling absorption. Laser-induced bulk damage in transparent material is described well by Wood (1986) and Kuzuu et al. (1999). Depending on the pulse duration of the laser, these mechanisms range from thermal effects to dielectric breakdown (Wood 1995). In addition, transparent materials are efficiently machined in the presence of absorbing liquids (Dolgaev et al. 1996; Ding et al. 2002)

Limiting ourselves to a qualitative approach and assuming almost perfect material (clear of inclusions, color centers, voids), we suggest that the thermal stress of the nozzle edge causes a rapid displacement in the sensitive region where the jet is produced (see Fig. 2). The laser spot can be approximated by assuming a gaussian laser beam. On the one hand, for zero off-center coupling, only the tail of the energy distribution overlaps with the nozzle edge. In this case, since the tail of the distribution only represents a fraction of the total energy, the coupling efficiency is expected to be small. On the other hand, a substantial amount of energy is coupled to the nozzle when off-center coupling is set (see illustration in Fig. 5).

\section{4}

Results

\section{1}

\section{Observation of successive disturbances}

An illustration of the laser-excited jet is presented in Fig. 6 for the following conditions: jet velocity $V_{j}=94 \mathrm{~m} / \mathrm{s}$, jet diameter $48 \mu \mathrm{m}$, laser average power $P=13 \mathrm{~W}$, and laser repetition rate $1 / \Delta t=20 \mathrm{kHz}$. The pulse energy is $0.7 \mathrm{~mJ}$, the pulse width is $317 \mathrm{~ns}$, and the peak power is $3 \mathrm{~kW}$.

The reconstructed image obtained with shadowgraphs reveals the evolution of the laser pulse-induced disturbances along the jet. To both observe the light scattered out of the jet and its shadowgraph contour (with high magnification), the flash emission is triggered on the laser pulse with a constant delay $d t 1$ corresponding to the laser period $\Delta t=50 \mu$ s. Successive disturbances (first between 20

\section{First disturbance}

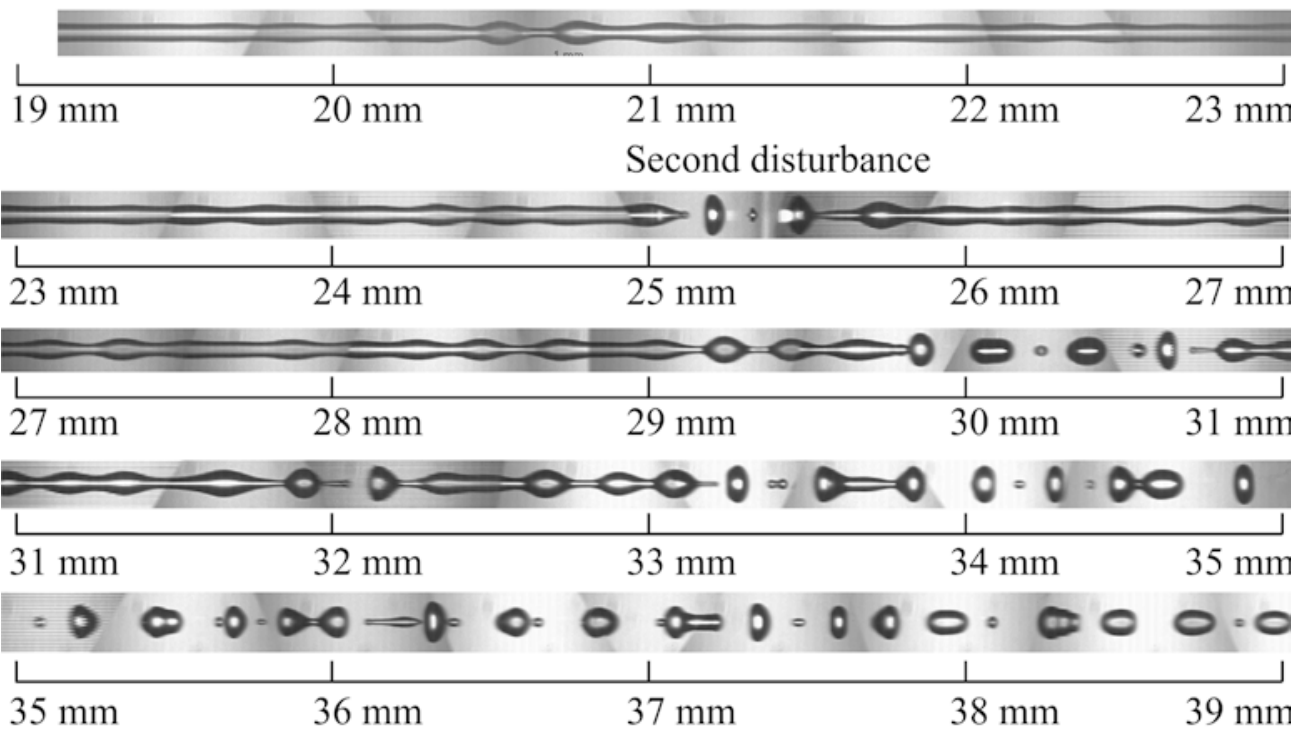

Fig. 6. Reconstructed image of laser-induced break-up obtained with the high-magnification system (see Fig. 3) with increasing distance from the jet origin and constant flash delay $d t 1=50 \mu \mathrm{s}$. Jet velocity $94 \mathrm{~m} / \mathrm{s}$, jet diameter $48 \mu \mathrm{m}$, laser average power $13 \mathrm{~W}$ at $20 \mathrm{kHz}$ 
and $21 \mathrm{~mm}$ distance) grow during their propagation until the break-up region where droplets are formed (at about $26 \mathrm{~mm}$ distance). Surface waves of small amplitude are observable between laser-generated disturbances. They correspond to the growth of natural initial perturbations. Since the delay between the laser pulse and the flash emission is kept constant, locations of observed disturbances are fixed along the jet. The presence of intense scattered green light from the second disturbance where the break-up already occurred is clearly observable.

Observation of the scattered light along the entire jet was achieved (see Fig. 7), where the green intensity profile is graphically presented. The two peaks in the figure reveal the presence of the two disturbances. A weak peak corresponds with the first disturbance because guided green light in the jet is partially scattered by the disturbance. An intense peak corresponds with the second disturbance because green light is totally scattered in the break-up region. The measured distance between the disturbances is equal to $25.5-20.8=4.7 \mathrm{~mm}$, which corresponds well with the expected distance $V_{\mathrm{j}} \times \Delta t$.

\section{2}

\section{Disturbance characteristics}

By setting a short variable delay $d t 1$ between the camera shutter and the laser pulse as well as shifting the camera axis along the jet, the evolution of the characteristics of the traveling disturbances is studied. The observation region is displaced so that the disturbance is in the field of view of the camera for the corresponding delay. This method allows the study of the progressive growth of the laser pulseinduced disturbances along the jet. The jet velocity is $94 \mathrm{~m} / \mathrm{s}$, the laser average power $20 \mathrm{~W}$. Examples of highmagnification shadowgraphs are presented in Fig. 8 with corresponding delay $d t 2$ and jet location $X$. The excellent repeatability in position and characteristics of the shadowgraphs was proven in numerous experiments. This demonstrates the constant phase relation between laser excitation and the laser disturbances. Moreover, the laser pulse-induced disturbances overbear the waves caused by the natural initial perturbations. Consequently, a nonfluctuating break-up is observed instead of the fluctuating one.

At the beginning of the jet, the amplitude of the disturbance is small (see image 1). Because of the growth of the propagating disturbance, the disturbance amplitude increases (image 2 and 3). In image 4, the break-up takes place and in image 5 droplets already appear.

Image processing is performed to obtain the jet contours corresponding to the different jet abscissa. The diameter of the jet versus jet abscissa is then calculated by subtracting the lower jet contour from the upper jet contour. Obtained diameter curves are superposed and shifted to the corresponding jet abscissa on the same graph shown in Fig. 9. The wavelength of disturbances is calculated by Fourier analysis of each diameter curve. Results show that up to the break-up point at $35.8 \mathrm{~mm}$, the main wavelength does not depend on the jet abscissa and is $220 \mu \mathrm{m}$. The
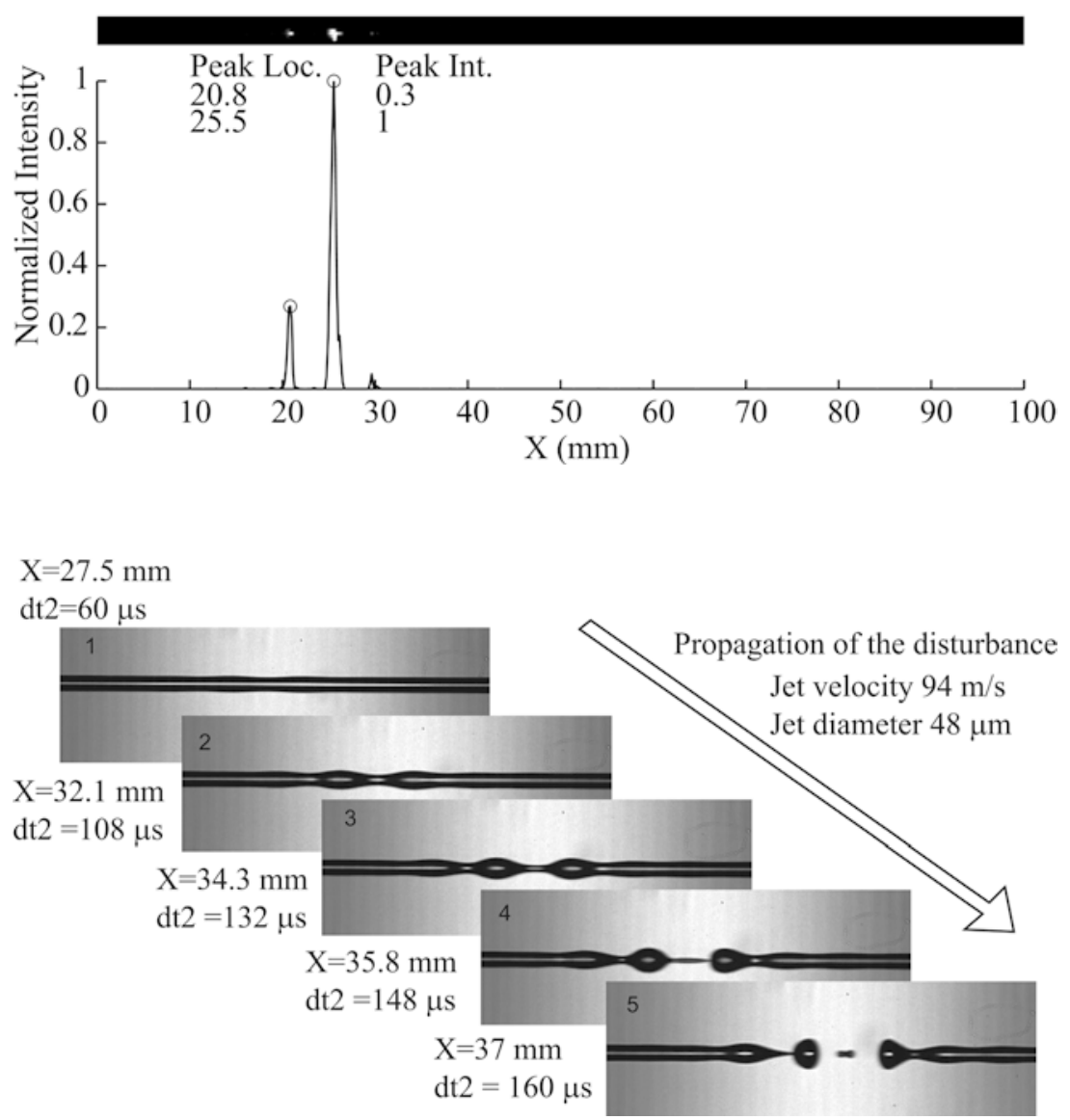

Fig. 7. Scattered green light. 10,000 pulses, $13 \mathrm{~W}$ at $20 \mathrm{kHz}, \mathrm{V}_{j}=94 \mathrm{~m} / \mathrm{s}$
Fig. 8. Series of jet observations triggered on laser pulse with a variable delay $d t 1$. Image 1 represents small disturbance amplitude.

Images 2 and 3 show the growing disturbance before the break-up. Image 4 shows break-up point. Image 5 shows the formed droplets. Jet velocity $94 \mathrm{~m} / \mathrm{s}$, jet diameter $48 \mu \mathrm{m}$, laser average power $20 \mathrm{~W}$ at $10 \mathrm{kHz}$ 


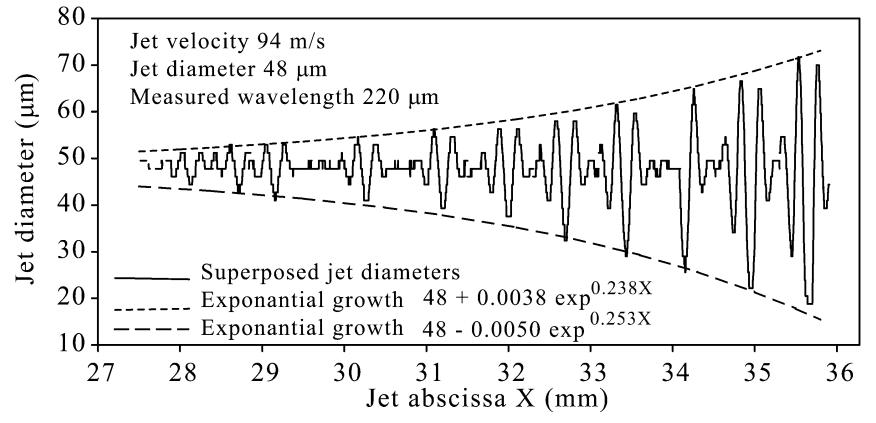

Fig. 9. Growth of the disturbance. Jet velocity $94 \mathrm{~m} / \mathrm{s}$, jet diameter $48 \mu \mathrm{m}$, laser average power $20 \mathrm{~W}$ at $10 \mathrm{kHz}$

main wavelength corresponds to the highest peak in the calculated Fourier spectra. To calculate the growth rate of the disturbance, both the lower and upper envelopes of the curves are interpolated using exponential functions, leading to the corresponding growth rates 0.253 and $0.238 \mathrm{~mm}^{-1}$.

\section{3}

\section{Comparison with theoretical predictions}

Calculations were done for water jet conditions identical to the experiments: velocity $V_{\mathrm{j}}=94 \mathrm{~m} / \mathrm{s}$ and jet radius $R_{\mathrm{j}}=24 \mu \mathrm{m}$. In terms of non-dimensional parameters, the jet can be defined by a Reynolds number of $R e=2,245$ and a Weber number of $W e=2,910$, where the Reynolds and Weber numbers are respectively defined by $V_{\mathrm{j}} R_{\mathrm{j}} / v_{1}$ and $\rho_{\mathrm{l}} R_{\mathrm{j}} V_{\mathrm{j}}^{2} / \sigma$, where $v_{l}, \rho_{l}$, and $\sigma$ are the water kinematic viscosity, the water density, and the water-air surface tension, respectively. The jet stability is also influenced by the viscosity ratio, $m=\mu_{g} / \mu_{\text {l }}$, and the density ratio, $\xi=\rho_{\mathrm{g}} / \rho_{\mathrm{l}}$, where the subscript 1 denotes quantities related to the liquid phase and $g$ the gas phase. For normal temperature conditions $\left(T=22^{\circ} \mathrm{C}\right)$, the corresponding values are $\xi=0.00123$ and $m=0.0178$.

As described in Sect. 2, the use of a sharp edged nozzle has several consequences. Instead of having a parabolic velocity profile over the jet cross-section, the profile is almost flat and the jet boundary does not touch the nozzle wall. Consequently, the ranges for the Reynolds and Weber numbers corresponding to different break-up types given in the literature (Lin and Reitz 1998) are not applicable for our case.

Therefore, we used a CFD software coupled with linear stability analysis to predict the jet behavior. The method consists of the calculation of a realistic basic flow for the liquid jet and the surrounding air and then the study of its linear stability by means of the classical small perturbation technique (see, e.g., Preziosi et al. 1989). The incompressible and laminar basic flow is computed using the finite-volume code Fluent (Fluent Inc., Lebanon, $\mathrm{NH}$, USA). Both liquid and gas flows are viscous and no heat transfer is considered. The conservation equations for mass, momentum, and all scalars are solved in Fluent using a cylindrical-coordinate formulation. A volume-offluid (VOF) model is used to track the liquid-gas interface.

Each physical quantity is written as a superposition of a mean value and of a fluctuating one and is introduced in

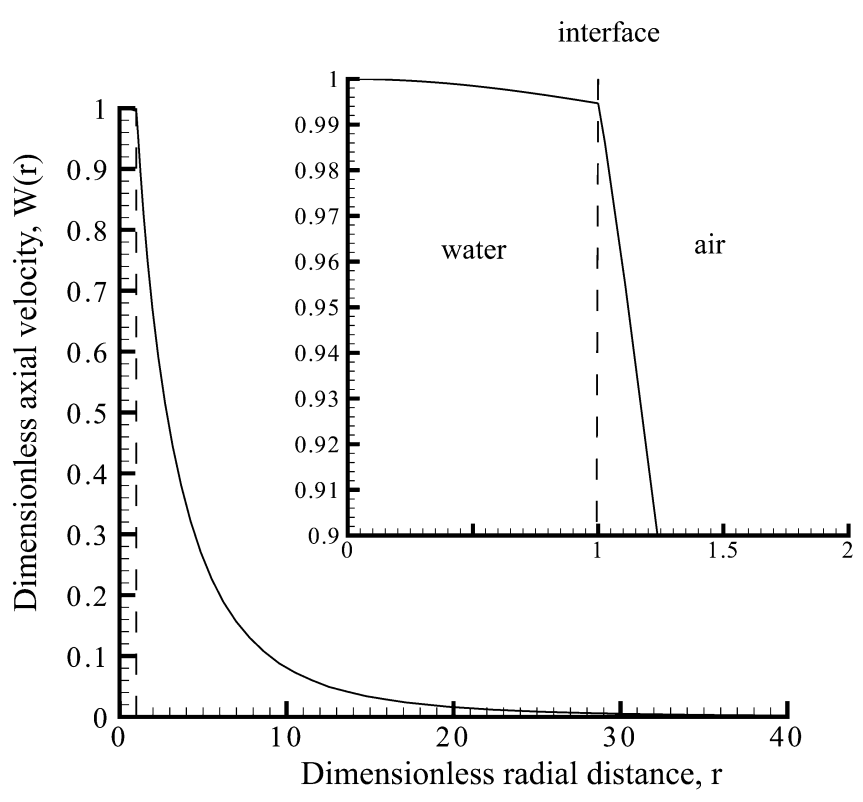

Fig. 10. Mean velocity distribution at a distance $x=28 \mathrm{~mm}$ from the nozzle. The velocity is normalized by the jet velocity $V_{j}=94$ $\mathrm{m} / \mathrm{s}$ and the radial component is normalized by the jet radius $D_{\mathrm{j}} / 2$. The upper graphic shows the interface region

the Navier-Stokes equations. The latter are linearized for the fluctuating value and the perturbation is searched with the normal mode mathematical form. In particular, the interface fluctuation is given by:

$\left.\eta(x, t)=\eta_{0} \exp [i(n \theta+k x)-i \omega t)\right]$,

where $\eta(x, t)$ represents the displacement of the interface from the position $r=1, \eta_{0}$ is the initial amplitude of the perturbation, and $(k, n)$ is the wave number vector, where $n$ is an integer equal to 0 for axisymetric perturbations. The axial wavenumber of the disturbance $k$ is related to the axial wavelength of the disturbance $\lambda$ by the relationship $k=2 \pi / \lambda$. The complex pulsation is $\omega=\omega_{\mathrm{r}}+i \omega_{i}$. The imaginary part $\omega_{i}$ represents the rate of growth $\left(\omega_{i}>0\right)$ or decay of the disturbance $\left(\omega_{i}<0\right)$, the real part $\omega_{\mathrm{r}}$ is $2 \pi$ times the disturbance frequency, and $\omega_{\mathrm{r}} / k$ is the wave propagation velocity of the disturbance in the direction of the liquid flow.

The stability equations are solved numerically by expanding the eigenfunctions in Chebyshev polynomials and employing a collocation algorithm; a method that is known to give very accurate eigenvalues for many stability problems using a minimal spectral resolution.

The mean velocity profiles corresponding to the water jet and entrained air flow are plotted in Fig. 10. As it has been discussed above, the velocity profile inside the jet is almost flat. One can note the large difference in the slopes of the velocity profiles in the liquid and the gas phases owing to the large difference in their viscosities.

Growth rates corresponding to an axisymmetric disturbance for the parameters given above are plotted in Fig. 11. One can see that the most amplified mode corresponds to the wavenumber $k=0.69$ and the corresponding growth rate is $\omega_{i}=0.00644$. As observed by Rayleigh (1878, 1879), this mode is characteristic of the capillary pinching 


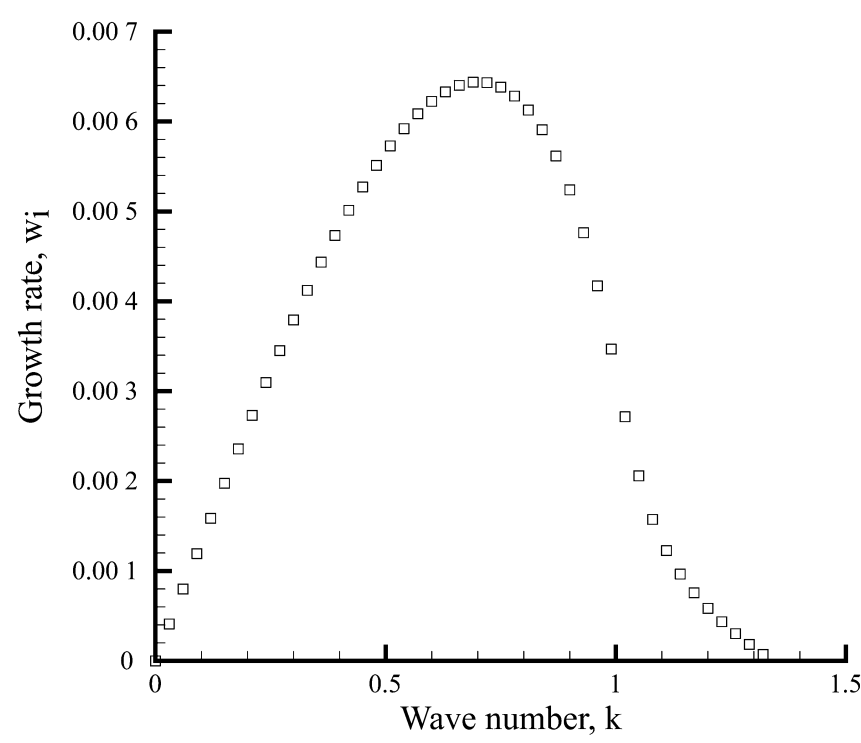

Fig. 11. Stability curve of a free water jet for $R e=2245, W e=2910$, $\xi=0.00123$, and $m=0.0178$

mechanism. The growth rate calculated with the Rayleigh theory gives 0.006572 .

The theoretical values can be compared to experimental results presented in the previous section. After normalization by the jet radius $R_{\mathrm{j}}=24 \mu \mathrm{m}$ and the jet velocity $V_{\mathrm{j}}=94 \mathrm{~m} / \mathrm{s}$, the measured wavelength $(220 \mu \mathrm{m})$ is $\lambda=9.17$, which corresponds to a wavenumber $k=0.69$. The mean value of the two growth rates calculated in Fig. 9, $0.245 \mathrm{~mm}^{-1}$, is then multiplied by the jet radius to obtain the non-dimensional growth rate $\omega_{i}=0.00589$. Therefore, the agreement between theoretical and experimental wavelength is very good (lower than $10 \%$ difference). The small discrepancy observed on the growth rate can be explained by the presence of non-linear effects that saturate the disturbance amplitude before the jet break-up (see Goedde and Yuen 1970). Obviously, the assumption of an exponential growth of the disturbance near the break-up point has to lead to a lower growth rate than the one predicted by a linear theory.

On the one hand, these results are readily identical to what can be found with the Rayleigh theory, which does not take into account the air flow around the jet and considers a constant velocity for the jet. On the other hand, in our calculations we are able to give the realistic velocity profile over the jet cross-section and the air flow is taken into consideration. Our conclusion is that for the case of sharp edged nozzle, the Rayleigh theory can be applied to an extended range of Reynolds and Weber numbers.

\section{4}

\section{Influence of the coupling}

By comparing the disturbances obtained with different coupling conditions for the same jet velocity and laser parameters it is shown that the laser-induced break-up strongly depends on the alignment of the focused laser spot with respect to the jet axis. Laser-generated disturbances are also compared to the natural initial disturbances. The jet velocity is set to $209 \mathrm{~m} / \mathrm{s}$, laser average power $13 \mathrm{~W}$ and repetition rate $20 \mathrm{kHz}$. The pulse energy is $0.7 \mathrm{~mJ}$, the pulse width is $317 \mathrm{~ns}$, and the peak power is $3 \mathrm{~kW}$. Applying the "light-guided method", statistical analysis of the disturbance locations along the jet is obtained by image processing of 50 visualizations of the entire jet with short exposures of $1 \mu$ s (50 laser pulses), and constant delay between the laser pulse and
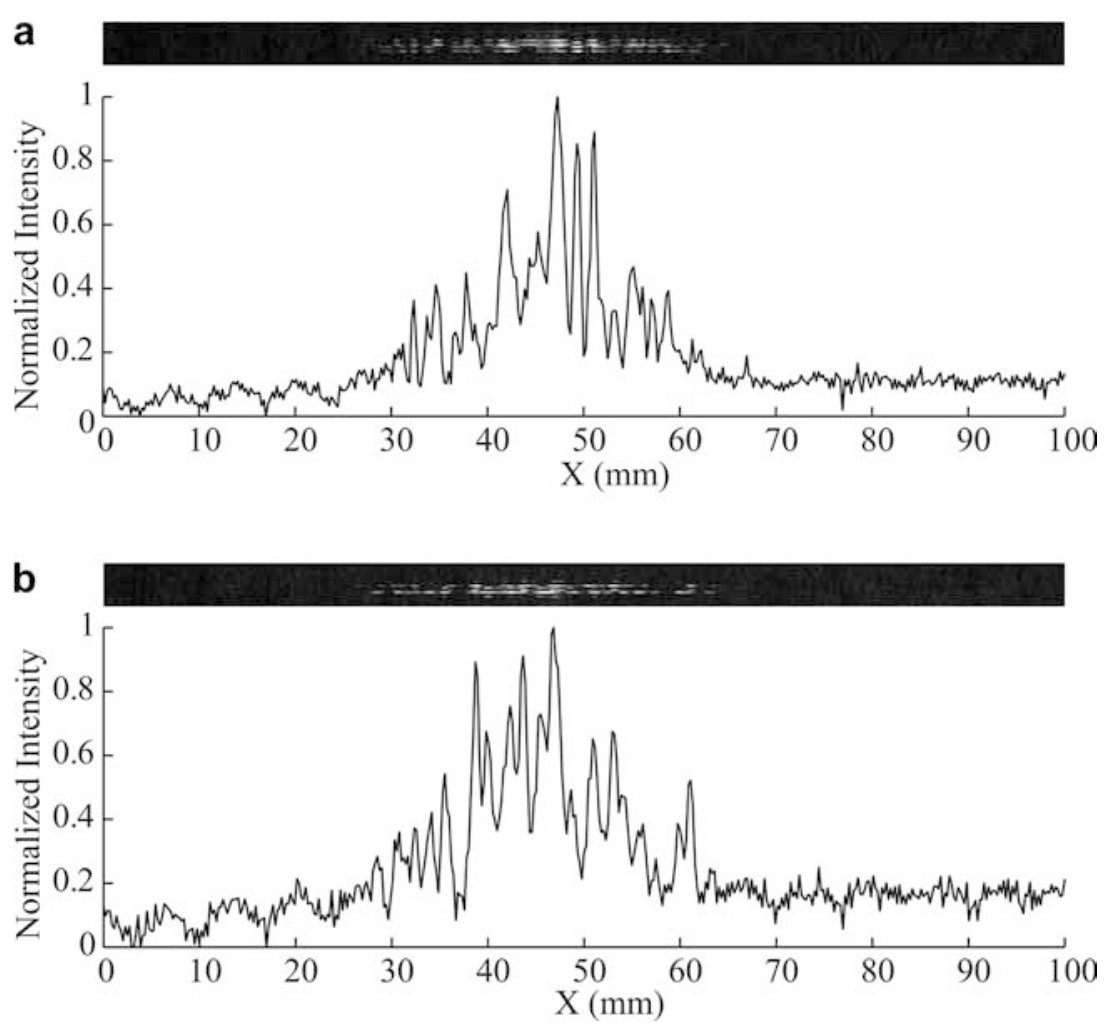

Fig. 12a, b. Intensity profile with focused laser spot, 50 pulses. Jet velocity $209 \mathrm{~m} / \mathrm{s}$. a Alignment power (few $\mathrm{mW}$ at $20 \mathrm{kHz}$ ). b Laser average power $13 \mathrm{~W}$ at $20 \mathrm{kHz}$ 


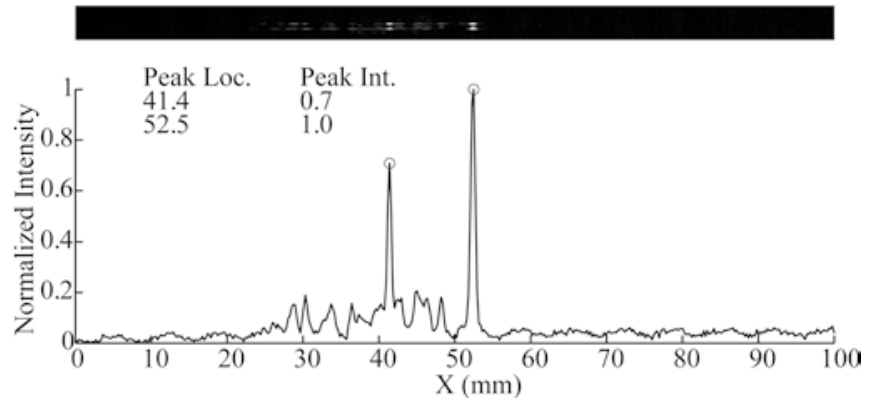

Fig. 13. Intensity profile with $9 \mu \mathrm{m}$ off-center coupling of the focused laser spot, 50 pulses. Jet velocity $209 \mathrm{~m} / \mathrm{s}$; laser average power $13 \mathrm{~W}$ at $20 \mathrm{kHz}$

visualization. Each image contains the light scattered out of the jet when the disturbance reached a minimum threshold amplitude.

In Fig. 12, the intensity profiles correspond to the focused laser spot centered into the jet with off-axis of $0 \mu \mathrm{m}$. Assuming a gaussian beam with $1 / e^{2}$ spot diameter $26 \mu \mathrm{m}$ passing through the centered circular aperture $60 \mu \mathrm{m}$ (the nozzle diameter), the beam power transmitted to the nozzle edge represents only $0.0025 \%$ of the total laser power.

In Fig. 12a, very low laser power is coupled into the jet (few $\mathrm{mW}$ ). This is achieved by attenuating the laser with the help of the attenuation block used for the alignment. The intensity profiles exhibit a random distribution of high intensity peaks caused by the fluctuations of the break-up. In Fig. 12b, the laser beam is not attenuated $(13 \mathrm{~W})$, and the obtained intensity profiles remain unchanged.

In Fig. 13, the laser average power is kept at $13 \mathrm{~W}$ but the focused laser spot is not centered into the nozzle, with an off-axis of $9 \mu \mathrm{m}$ in comparison to the jet radius of $24 \mu \mathrm{m}$. This is equivalent to a $37.5 \%$ relative off-axis and the beam power transmitted to the nozzle edge approximately represents $0.5 \%$ of the total laser power. As a result, distinguishable peaks clearly appear in the intensity profile owing to the successive laser pulses. Consequently, the laser pulse-induced break-up is obtainable when the laserfocused spot is not centered in the nozzle.

By comparing the graphics in Figs. 12 and 13, we observe that laser pulses lead to different break-up characteristics when the alignment coupling was changed for the same average power, peak power, and repetition rate of the laser. On the one hand, when the focused laser spot is well centered, little light is focused on the edge of the nozzle so that the nozzle does not induce disturbance on the jet. Moreover the possible hot source caused by water absorption is centered with respect to the jet, resulting in an initial and weak symmetric hydrodynamic disturbance that has a negligible effect for the jet stability. On the other hand, when off-center coupling is set, more light is absorbed by the surface of the nozzle leading to local heat and jet destabilization. Also, the hot source caused by water absortion closer to the jet interface can result in a non-symmetric disturbance with a higher initial amplitude.

\section{Conclusions}

The laser pulses generate a series of disturbances traveling at jet speed. The disturbance grows until the disruption of the jet is completed. During this time, other laser pulses can be coupled into the jet. Consequently, successive traveling and growing disturbances are present on the jet. Instead of having a fluctuating break-up length, a betterdefined break-up length is obtained when laser pulses excite the jet. The measured wavelength and growth rate of laser-generated disturbances correspond well to the ones predicted by the linear stability theory of free jets. Moreover, the excited mode corresponds to the capillary pinching mechanism. The control of the jet is not only sensitive to the laser peak power but also to the coupling of the laser beam in the jet waveguide. We observed a possible control of the jet stability even for low peak power if the coupled laser beam is misaligned with respect to the nozzle center.

\section{References}

Collicott SH, Zhang S, Schneider SP (1994) Quantitative liquid jet instability measurement system using asymmetric magnification and digital image processing. Exp Fluids 16:345-348

Ding X, Kawagushi Y, Niino H, Yabe A (2002) Laser-induced highquality etching of fused silica using a novel aqueous medium. Appl Phys A 75:641-645

Dolgaev SI, Lyalin AA, Simakin AV, Shafeev GA (1996) Fast etching of sapphire by a visible range quasi-cw laser radiation. Appl Surf Sci 96:491-495

Donnelly RJ, Glaberson W (1966) Experiments on the capillary instability of a liquid jet. Proc R Soc Lond Ser A, Math Phys Sci 290:547-556

Goedde EF, Yuen MC (1970) Experiments on liquid jet instability. J Fluid Mech 40:495-511

Grant RP, Middleman S (1966) Newtonian jet stability. AIChE J 12:669-678

Kuzuu N, Yoshida K, Yoshida H, Kamimura T, Kamisugi N (1999) Laser induced bulk damage in various types of vitreous silica at 1064, 532, 355 and $266 \mathrm{~nm}$ : evidence of different damage mechanisms between $266 \mathrm{~nm}$ and longer wavelengths. Appl Opt $38: 2510-2515$

Lafrance P (1975) Nonlinear breakup of a laminar liquid jet. Phys Fluids 18:428-432

Leroux S, Dumouchel C, Ledoux M (1996) The stability curve of Newtonian liquid jets. Atom Sprays 6:623-647

Lin S, Reitz RD (1998) Drop and spray formation from a liquid jet. Annu Rev Fluid Mech 30:85-105

Noack J, Hammer DX, Noojin GD, Rockwell BA, Vogel A (1998) Influence of pulse duration on mechanical effects after laser-induced breakdown in water. J Appl Phys 83(12):7488-7495

Preziosi L, Chen K, Joseph DD (1989) Lubricated pipelining: stability of core-annular flow. J Fluid Mech 201:323-356

Rayleigh, Lord (1878) On the instability of jets. Proc Lond Math Soc 10:4-13

Rayleigh, Lord (1879) On the capillary phenomena of jets. Proc R Soc 29:71-97

Richerzhagen B (2001) Chip singulation process with a water-jet guided laser. Solid State Technol 44(4):25-28

Rutland DF, Jameson GJ (1971) A non-linear effect in the capillary instabillity of liquid jets. J Fluid Mech 46:267-271

Spiegel Á, Vágó N, Wagner FR (2004) High efficiency Raman scattering in micro water jets. Opt Eng 43(2):450-454

Sterling AM, Sleicher CA (1975) The instability of capillary jets. J Fluid Mech 68(3):477-495

Taub HH (1976) Investigation of nonlinear waves on liquid jets. Phys Fluids 19:1124-1129 
Vágó N, Spiegel Á, Couty P, Wagner FR, Richerzhagen B (2003) New technique for high-speed microjet breakup analysis. Exp Fluids 35:303-309

Vogel A, Lauterborn W, Timm R (1989) Optical and acoustic investigations of the dynamics of laser-produced bubbles near a solid boundary. J Fluid Mech 206:299-338

Wood RM (1986) Laser damage in optical materials. Adam Hilger Press, Bristol
Wood RM (1995) Pulse duration dependance of laser damage mechanisms revisited. SPIE 2428:531-545

Xing JH, Boguslawski A, Soucemarianadin A, Atten P, Attanë P (1996) Experimental investigation of capillary instability: results on jet stimulated by pressure modulations. Exp Fluids 20:302-313 